\title{
Fatal Case of Spontaneous Rectus Sheath Hematoma Caused by Anticoagulant and Steroid Therapy during Hospital Admission
}

\author{
Ivan Romic ${ }^{a} \quad$ Goran Pavlek $^{a} \quad$ Marko Mance $^{a} \quad$ Marijan Romic $^{b} \quad$ Trpimir Moric $^{a}$ \\ ${ }^{a}$ Department of Surgery, University Hospital Centre Zagreb, Zagreb, Croatia; \\ b Department of Surgery, University Hospital 'Sestre milosrdnice', Zagreb, Croatia
}

\section{Keywords}

Hematoma · Abdominal wall · Anticoagulation

\section{Summary}

Background: Rectus sheath hematoma (RSH) is a rare but potentially dangerous clinical entity that requires medical supervision. Case Report: Here we discuss one such case which describes the fatal course of spontaneous RSH during hospital admission. Conclusion: Usually, $\mathrm{RSH}$ presents as a minimal abdominal wall swelling with self-limiting course but in the case of hematoma and size progression, specific conservative or invasive measures are necessary to prevent serious complications including hypovolemic shock and death.

(C) 2018 S. Karger GmbH, Freiburg

\section{Introduction}

Spontaneous rectus sheath hematoma (RSH) is usually a result of anticoagulation therapy, and conservative treatment is sufficient in the majority of cases. Abdominal muscle straining (coughing, sneezing, or exercise) may be a precipitating factor but may also be absent in many cases.

The conservative approach consists of interruption of anticoagulation and antiaggregation therapy, correction of coagulation parameters, and blood volume restoration. Beside anticoagulation therapy, some other predisposing and contributing factors can be named and include e.g. obesity, corticosteroid therapy, arterial hypertension, pregnancy, previous abdominal surgeries, and thrombophilia [1].
For larger hematomas, a hospitalization is required so that complications and signs of hematoma expansion can be detected early in its course, which is essential for adequate therapy. Signs of hemodynamic instability, intraperitoneal rupture, or intra-abdominal organ compression with compartment syndrome necessitate an invasive approach, i.e. surgical hemostasis or arterial embolization.

Computed tomography (CT) is the gold standard in the diagnosis of RSH and may help to differentiate RSH from other intra-abdominal pathologies. Ultrasound may be useful as a part of further follow-up examinations $[1,2]$.

\section{Case Presentation}

A 51-year-old woman with a history of permanent atrial fibrillation, Hashimoto's disease, and arterial hypertension was admitted to the Rheumatology Department for worsening right shoulder and bilateral wrist pain associated with high-grade fever $\left(39^{\circ} \mathrm{C}\right)$ and elevated inflammatory markers (leukocytosis $22 \times 10^{9} / 1$ and C-reactive protein $326 \mathrm{mg} / \mathrm{l}$ ). After bone scintigraphy and X-ray imaging the diagnosis of polymyalgia rheumatica was established. International normalized ratio was 0.9 despite Martefarin therapy, partial thromboplastin time (PTT) was $75 \mathrm{~s}$, and thrombocyte count was $230,000 \mu \mathrm{l}$. In addition, right leg swelling was noted and Doppler ultrasound revealed deep vein thrombosis below the popliteal trifurcation. During the 6 days of hospitalization, the patient received anti-inflammatory steroid therapy (prednisone), antibiotic therapy (clindamycin and ciprofloxacin), and subcutaneous (right and left shoulder) injections of therapeutic enoxaparin $(2 \times 60 \mathrm{mg})$. Antibiotics were given to prevent the development of septic arthritis (high fever and a high level of inflammatory markers could have been a sign of it). No signs of septic thrombocytopenia were detected (thrombocyte count was 200,000-250,000 per $\mu$ l of blood). In the morning of day 7 , she developed a large visible and palpable abdominal wall hematoma in the right lower abdominal quadrant. Abdominal CT was done and revealed an RSH of $8.2 \times 3 \times 6.3 \mathrm{~cm}$ located in the right lower quadrant abdominal wall, without active contrast extravasation and without intraperitoneal blood detection (fig. 1, 2). Physical examination and CT scan did not reveal other hematomas, and the coagulation parameters at this time were as follows: PTT 105 s and thrombocyte count 210,000 per $\mu$ l of blood.

\section{KARGER}

() 2018 S. Karger GmbH, Freiburg 
The hemoglobin level was $84 \mathrm{~g} / \mathrm{dl}$; the patient had stable vital signs, however, so our initial approach was based on conservative measures that included low molecular heparin and steroid interruption, opioid painkillers, ice packs, and abdominal wall compression. Also, tranexamic acid, 2 units of fresh frozen plasma (FFP), and 4 units of packed red blood cells (PRBC) were administered. Since the low hemoglobin value persisted even after PRBC transfusions, we performed selective percutaneous angiography with intention to carry out arterial embolization, but there was no detectable active bleeding from the inferior epigastric artery or its branches.

However, during the next $8 \mathrm{~h}$, the patient's hemoglobin level dropped to 72 $\mathrm{g} / \mathrm{dl}$, and the blood pressure was $90 / 60$ with tachycardia of 130 . The hematoma was becoming visibly larger. She was transferred to the intensive care unit for resuscitation and monitoring. Over the next few hours, her vital functions were stabilized with fluid resuscitation and oxygen, but the hemoglobin level could not be kept above $80 \mathrm{mg}$ even after 6 more units of PRBC and 2 FFP units.

At this point, the patient was offered surgical therapy but refused it, and even after psychiatric assessment and consultation she signed an informed refusal form. After four more PRBC transfusions and $16 \mathrm{~h}$ after the onset of symptoms, the patient became hemodynamically unstable; however, she refused intubation and surgery again so we performed another CT scan that revealed progression of abdominal wall hematoma which was now compressing intra-abdominal structures, filling the whole pelvic cavity, and reaching the level of the right costal margin. Furthermore, a moderate amount of free intraperitoneal blood was seen. Soon after, the patient suffered respiratory and cardiac arrest with return of spontaneous circulation after $10 \mathrm{~min}$ of cardiopulmonary resuscitation. Since she was intubated and unconscious, her family approved further surgical measures and the patient was taken to the operating room for surgical hemostasis and hematoma evacuation.

Intraoperatively, after the anterior rectal muscle fascia was opened, a surgeon found and evacuated 41 of partly coagulated hematoma extraperitoneally and 1 more liter of hematoma intraperitoneally. There was no active bleeding visible. Hemostasis in the interfascial space was achieved by packing with gauzes, but at the end of the procedure the patient suffered cardiac arrest again and died of hemorrhagic shock despite intensive resuscitation measures.

\section{Discussion}

Our article presents a rare case of spontaneous $\mathrm{RSH}$ which was closely followed and treated under medical supervision; because of the patient's refusal of surgical therapy, however, it had a fatal outcome. There are very few scientific articles about the surgical management of RSH, since the majority of cases is treated non-surgically, but our case proves that hematoma may expand and lead to hemodynamic instability and death within $24 \mathrm{~h}$ after onset, despite aggressive resuscitative measures. We cannot know for sure if anticoagulant and steroid therapy had an impact on RSH development in this case, but we can confirm that there was no abdominal trauma and that no enoxaparin injections were given directly into abdominal wall.

Our initial and follow-up abdominal CT scan objectivized the hematoma progression as well as the development of intraperitoneal rupture and abdominal compartment syndrome.

Berná et al. [3] suggested a classification of RSH into three types based on findings observed in CT scan. Type 1 hematoma is intramuscular as well as unilateral and does not dissect along the fascial planes. Type 2 is intramuscular though with blood between the muscle and the transversalis fascia. It may be unilateral but is usually bilateral, and no blood is observed occupying the prevesical space. In type 3 hematoma, the blood is observed between the

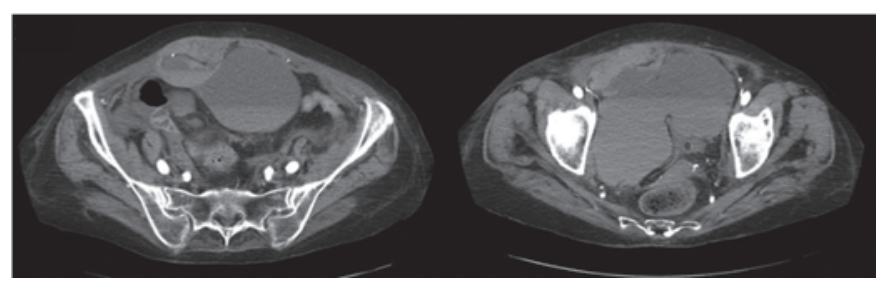

Fig. 1. Contrast axial computed tomography scan images of lower abdomen and pelvis showing the rectus sheath hematoma sized $8.2 \times 3 \times 6.3 \mathrm{~cm}$. Fluid collections of different density suggest active bleeding.

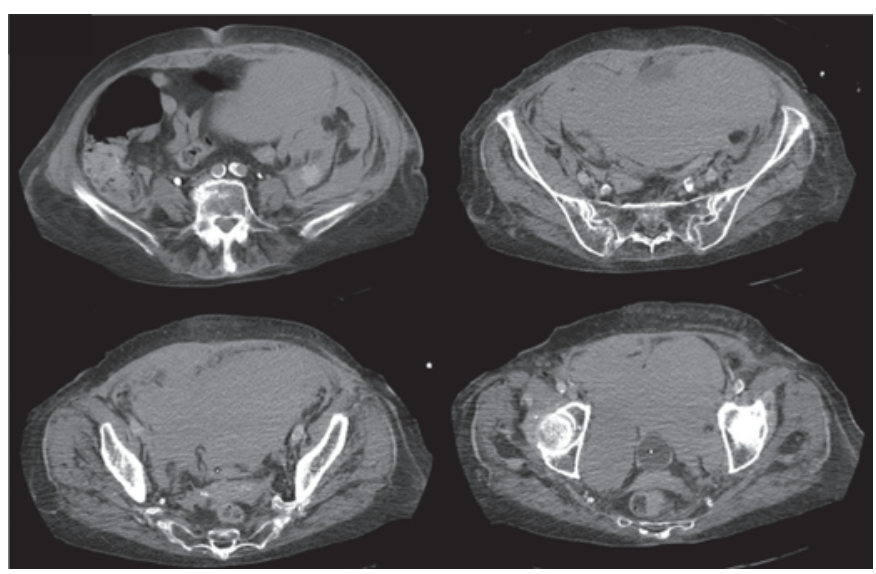

Fig. 2. Computed tomography scan images in the same patient $24 \mathrm{~h}$ later; there is a significant progression in size, and the hematoma has now extended above the umbilicus level and occupies the whole pelvic cavity.

transversalis fascia and the muscle, in the peritoneum, and in the prevesical space. A hematocrit effect can be observed, and, on occasion, hemoperitoneum is produced. Type 1 and 2 may require hospitalization and (though less common) blood transfusions; however, most patients are discharged to home within 5 days, and invasive therapy is almost never required. Type 3 often requires transfusions, but only rarely hemodynamic instability may develop that cannot be controlled with FFP and fluid resuscitation; such unstable patients may require surgical intervention.

There are no clear guidelines about the therapeutic approach in $\mathrm{RSH}$; nevertheless, individual clinical decision making should be based on the presence of some alarming signs and symptoms such as: enlarging hematomas, hemodynamic instability unresponsive to fluid resuscitation, signs of peritoneal irritation, refractory pain, and persistent gastrointestinal or urinary symptoms [2, 4].

Other factors which should be considered include the cause, location size and progression of hematoma, general condition and comorbidities of the patient, coagulation status, hematocrit stabilization after blood transfusions, and availability of an interventional radiologist. We consider hemodynamic instability and abdominal compartment syndrome as absolute indications for surgical therapy. In other cases, the initial conservative approach is justified and consists of removal of predisposing factors, blood transfusions, coagulation correction, icing, external hematoma compression, pain management, and blood pressure regulation [5]. 
Since the number of RBC units administered is not established as a value that helps to define indications for an invasive approach, we suggest that if the patient remains unstable after 6-8 PRBC units within $24 \mathrm{~h}$, an invasive approach should be considered not only because of the risk of bleeding progression but also because of transfusion-related acute lung injury.

There are two invasive modalities, i.e. therapeutic angiography with embolization of the bleeding vessel, which became the current treatment of choice, and operative therapy with hematoma evacuation, ligation of bleeding vessels, and closed-suction drainage. Invasive therapy has a high success rate, and recurrences following such therapy have not been reported $[1,6]$. and may not be successfully managed with a conservative approach, even if it is performed in a hospital where all medical and intensive care measures are available. If there is no adequate clinical and hematocrit response on recoagulation and PRBC transfusions, a clinician should have a low threshold for invasive approach since a severe hemodynamic compromise with associated mortality may develop in these patients.

\section{Disclosure Statement}

There is no conflict of interest for any of the authors.

\section{Conclusion}

In most cases an RSH is a self-limited condition; however, our case suggests that some RSHs may take an unpredictable course

\section{References}

1 Mendoza Moreno F, Díez Alonso M, Villeta Plaza R, et al: Spontaneous haematoma of the anterior rectus abdominis muscle (Article in English, Spanish). Cir Esp 2016;94:294-299.

2 Hatjipetrou A, Anyfantakis D, Kastanakis M: Rectus sheath hematoma: a review of the literature. Int J Surg 2015;13:267-271.
Berná JD, Garcia-Medina V, Guirao J, Garcia-Medina J: Rectus sheath hematoma: diagnostic classification by CT. Abdom Imaging 1996;21:62-64.

4 Sullivan LEJ, Wortham DC, Litton KM: Rectus sheath hematoma with low molecular weight heparin administration: a case series. BMC Res Notes 2014;7:586.
Ortega-Carnicer J, Ceres F: Rectus sheath haematoma with severe haemodynamic compromise after enoxaparin use for unstable angina. Resuscitation 2003;57: 113-114.

6 James RF: Rectus sheath haematoma. Lancet 2005;365: 1824. 